\title{
Changes in the serum proteome associated with the development of hepatocellular carcinoma in hepatitis C-related cirrhosis
}

\author{
DG Ward', Y Cheng', G N'Kontchou', TT Thar', N Barget ${ }^{2}$, W Wei', LJ Billingham', A Martin', M Beaugrand ${ }^{2}$ \\ and PJ Johnson ${ }^{*, I}$ \\ 'Cancer Research UK Institute for Cancer Studies, School of Medicine, University of Birmingham, Edgbaston, Birmingham BI5 2TT, UK; \\ ${ }^{2}$ Hepto-gastroenterology and Pathology Department, Jean Verdier Hospital, Assistance Publique-Hospitaux de Paris, UPRES EA 3409, UFR SMBH, \\ Université Paris 13, Bondy, France
}

\begin{abstract}
Early diagnosis of hepatocellular carcinoma (HCC) is the key to the delivery of effective therapies. The conventional serological diagnostic test, estimation of serum alpha-fetoprotein (AFP) lacks both sensitivity and specificity as a screening tool and improved tests are needed to complement ultrasound scanning, the major modality for surveillance of groups at high risk of HCC. We have analysed the serum proteome of 182 patients with hepatitis C-induced liver cirrhosis (77 with HCC) by surface-enhanced laser desorption/ionisation time-of-flight mass spectrometry (SELDI). The patients were split into a training set (84 non-HCC, $60 \mathrm{HCC}$ ) and a 'blind' test set $(21$ non-HCC, $17 \mathrm{HCC}$ ). Neural networks developed on the training set were able to classify the blind test set with $94 \%$ sensitivity (95\% Cl $73-99 \%$ ) and $86 \%$ specificity (95\% Cl 65-95\%). Two of the SELDI peaks (23/23.5 kDa) were elevated by an average of $50 \%$ in the serum of HCC patients $(P<0.00 \mathrm{I})$ and were identified as $\kappa$ and $\lambda$ immunoglobulin light chains. This approach may permit identification of several individual proteins, which, in combination, may offer a novel way to diagnose HCC. British Journal of Cancer (2006) 94, 287-292. doi: I 0. I038/sj.bjc.6602923 www.bjcancer.com
\end{abstract}

Published online 10 January 2006

(c) 2006 Cancer Research UK

Keywords: hepatocellular carcinoma; serum; proteome; SELDI

Hepatocellular carcinoma (HCC) is the fifth commonest cancer in the world today and the overall 5-year survival rate remains less than 5\% (Parkin et al, 2001). Although the incidence rate is likely to fall with the institution of mass vaccination against the hepatitis B virus, initiated in the 1980s (Chang et al, 1997), this will not have a major impact for many years as the age of presentation is over 50 years in most areas of the world. Furthermore, there is no prospect of a vaccine against the hepatitis $C$ virus, the major aetiological factor for HCC in the US, Japan and Southern Europe (Di Bisceglie et al, 1991; El-Sarag and Hashem, 2004). Despite the absence of randomised clinical trials, there is strong evidence that surgical resection, liver transplantation or ablative therapies significantly improve survival (Bruix et al, 2001; Beaugrand et al, 2005). Such approaches are, however, only applicable to those in whom the tumour is detected at an early stage, typically less than $3 \mathrm{~cm}$ in diameter without vascular involvement and tumours only rarely present with symptoms at this stage (Mazzaferro et al, 1996; Bruix et al, 2001; Beaugrand et al, 2005). Early diagnosis has, therefore, become a priority. Surveillance of high-risk groups, such as those with cirrhosis, has been shown to permit detection of small tumours and there is emerging evidence that this is associated with improved survival (Sherman, 2005). Currently, surveillance involves both serological testing with serum alpha-fetoprotein

*Correspondence: Professor PJ Johnson; E-mail: p.johnson@bham.ac.uk Received 15 August 2005; revised 21 November 2005; accepted 29 November 2005; published online 10 January 2006
(AFP) estimation and ultrasound scanning, typically at 6 monthly intervals (Mazzaferro et al, 1996; Trevisani et al, 2002). However, while AFP may be a useful diagnostic serum marker in patients with advanced symptomatic disease, it is much less useful in patients with earlier/small tumours where its sensitivity is low (Mazzaferro et al, 1996; Johnson, 2001; Trevisani et al, 2002; Sherman, 2005).

Ultrasound is far more sensitive (in the order of $80 \%$ ) but it is highly operator dependent (Trevisani et al, 2002; Sherman, 2005). More specific and sensitive serological tests, to complement ultrasound scanning, would be of great clinical value.

Surface-enhanced laser desorption/ionisation time-of-flight mass spectrometry (SELDI) has shown potential for cancer biomarker discovery (Adam et al, 2002; Li et al, 2002; Chen et al, 2004; Zhang et al, 2004). A subset of the proteome of a biological sample, such as serum, which binds to a specific solidphase chromatographic surface (known as a 'protein-chip array'), is subsequently ionised and detected by time-of-flight mass spectrometry. The peak intensities in the SELDI spectra reflect the abundance of proteins and peptides in the serum. The technique is relatively high throughput, allowing samples to be processed in 96-well format at a rate of up to several hundred serum analyses per day per analyst. Various computer-based pattern recognition approaches can then be applied to discriminate between patient groups, for example, those with and without a particular cancer.

SELDI technology has been applied to identify potential serological diagnostic markers for several cancers including 
ovarian cancer (Zhang et al, 2004), prostate cancer (Adam et al, 2002), breast cancer (Li et al, 2002) and colon cancer (Chen et al, 2004). Published studies have indicated that the SELDI approach may also be used to diagnose HCC (Poon et al, 2003; Paradis et al, 2005; Schwegler et al, 2005), although only Paradis et al (2005) used an independent test set and gave details on experimental reproducibility. All three studies were based on small numbers of patients with either undefined or late-stage HCC or chronic liver disease arising from several different aetiologies. In addition, there has been little consensus on the proteomic features that are significantly different in the serum of HCC patients.

The present study was confined to patients with cirrhosis and hepatitis $\mathrm{C}$ infection, as hepatitis $\mathrm{C}$ infection is the most common cause of cirrhosis in the western world, carrying a high risk of HCC. In addition, we chose to study patients with small HCC $(3 \mathrm{~cm}$ mean diameter, range $1-11 \mathrm{~cm}$ ) in order to detect early changes that could be usable in a screening situation. Being cognisant of the controversies that surround the use of SELDI technology to identify biomarkers (Baggerly et al, 2004; Diamandis, 2004; Ransohoff, 2004), we were particularly careful to address issues of reproducibility and validity. We used a 'training' data set to develop artificial neural networks that permitted classification of patients as HCC or non-HCC and then applied these networks to a 'blind' test data set. In addition, we purified and identified two of the most discriminatory proteomic features with $\mathrm{m} / \mathrm{z}$ ratios of 23000 and 23500 .

\section{MATERIALS AND METHODS}

\section{Sample collection}

Serum samples were collected between May 1994 and January 2005 at Jean Verdier Hospital, Bondy, France. Sample collection was officially registered and all patients gave informed consent. Sera were stored at $-80^{\circ} \mathrm{C}$. All patients tested positive for hepatitis $\mathrm{C}$ antibodies and hepatitis C RNA on the day of sampling. Hepatocellular carcinoma was diagnosed histologically or noninvasively, according to the Barcelona criteria (Bruix et al, 2001). Samples were transported on dry ice to the University of Birmingham, UK, for analysis in February 2005, defrosted (on ice) and multiple $20 \mu \mathrm{l}$ aliquots taken and stored at $-80^{\circ} \mathrm{C}$ pending SELDI analysis. Quality control (QC) samples were prepared by mixing equal volumes of serum from 27 healthy individuals and stored as multiple aliquots at $-80^{\circ} \mathrm{C}$.

\section{Study design}

The study consisted of 84 non-HCC patients and 60 HCC patients (the training set) and 38 samples where the classification of HCC/ non-HCC identities was not revealed to the UK-based proteomics team (the blind test set) (Table 1). Independent duplicate SELDI spectra were collected for all serum samples using $\mathrm{Cu}^{2+}$-loaded IMAC30 protein-chip arrays. Samples were processed using six 96-well bioprocessors over a 2-week period. Three spots per bioprocessor were devoted to identical QC samples, one spot to a $0-20 \mathrm{kDa}$ calibration mix and one spot to a $20-200 \mathrm{kDa}$ calibration mix. Block randomisation was utilised: to the eight spots on each protein-chip array, we applied in random sequence three serum samples from patients without HCC, three serum samples from patients with HCC and two serum samples from the blind test set or one serum sample from the blind test set and either one QC sample or calibration mix. All samples were analysed once on bioprocessors $1-3$ and then a separate aliquot of each patient's serum was analysed a second time on bioprocessors $4-6$, ensuring that the measurement duplicates were not processed on the same day.
Table I Patient demographics

\begin{tabular}{lccc}
\hline Patient group & No. patients & Male/female & Mean age (s.d.) \\
\hline Non-HCC (training set) & 84 & $44 / 40$ & $65.4(11.7)$ \\
HCC (training set) & 60 & $41 / 19$ & $71.8(15.3)$ \\
Non-HCC (test set) & 21 & $8 / 13$ & $67.4(12.2)$ \\
HCC (test set) & 17 & $7 / 10$ & $68.1(10.0)$ \\
\hline
\end{tabular}

The table shows the number, sex and mean age of the patients analysed in this study. $\mathrm{HCC}=$ hepatocellular carcinoma

\section{Surface-enhanced laser desorption/ionisation procedure}

An initial experiment using pooled sera from HCC and non-HCC patients was conducted to decide whether H50, CM10, Q10 or $\mathrm{Cu}^{2+}$-loaded IMAC30 protein-chip arrays were best able to detect changes in the serum proteome characteristic of HCC. The $\mathrm{Cu}^{2+}$ loaded IMAC30 performed best both in terms of the total number of peaks detected and the number of peak intensities that were significantly different between the HCC and non-HCC pooled samples. We proceeded to analyse all of the patient's samples in duplicate using $\mathrm{Cu}^{2+}$-loaded IMAC30 protein-chip arrays. The protein-chip arrays were placed in a 96-well bioprocessor and prepared by a $5 \mathrm{~min}$ incubation with $50 \mu \mathrm{l}$ of $100 \mathrm{mM} \mathrm{CuSO}_{4}$ followed by a water rinse and $3 \times 10 \mathrm{~min}$ equilibrations with $200 \mu \mathrm{l}$ of binding buffer $\left(100 \mathrm{~mm} \mathrm{NaCl}, 500 \mathrm{~mm} \mathrm{NaH} \mathrm{PO}_{4} / \mathrm{NaOH}(\mathrm{pH}\right.$ 7.0)). Serum samples were defrosted on ice and diluted five-fold with $9 \mathrm{M}$ urea, $2 \%$ CHAPS, $50 \mathrm{~mm}$ Tris/ $\mathrm{HCl}$ ( $\mathrm{pH}$ 9.0). Following a brief vortex, the samples were left on ice for $30 \mathrm{~min}$ prior to a 10 fold dilution in binding buffer. These 50 -fold final dilution samples were loaded on the bioprocessor $(100 \mu \mathrm{l}$ per spot) and incubated at room temperature for $1 \mathrm{~h}$ with shaking at 900 r.p.m. After this period, the nonbound material was discarded and the protein-chip arrays were washed $(4 \times 10 \mathrm{~min}$ incubations with $200 \mu \mathrm{l}$ of binding buffer followed by a water rinse). The protein-chip arrays were allowed to dry for $30 \mathrm{~min}$ prior to addition of $1 \mu \mathrm{l}$ of $50 \%$ saturated sinapinic acid in $50 \%$ acetonitrile, $0.5 \%$ trifluoroacetic acid. The spots were then allowed to dry for another $30 \mathrm{~min}$ prior to a second $1 \mu \mathrm{l}$ addition of sinapinic acid. The protein-chip arrays were analysed in a PBS IIc protein chip reader equipped with an autoloader (Ciphergen, UK). Spectra were collected over 0-20 and $0-200 \mathrm{kDa}$ ranges (488 laser shots) using laser intensities of 165 and 210 , respectively. Spectra were externally calibrated in the $0-$ $20 \mathrm{kDa}$ range using all-in-one peptide standard (Ciphergen) with added cytochrome $c$ and myoglobin (Sigma). The $0-200 \mathrm{kDa}$ range was calibrated using chymotrypsinogen, bovine serum albumin and phosphorylase b (Sigma). Spectra were normalised using the total ion current from 2 to 20 and 20 to $200 \mathrm{kDa}$. Peaks were selected and clustered using Biomarker Wizard software (Ciphergen) with the signal to noise ratio $>5$ for the first pass and $>2$ for the second, a cluster mass window of $0.2 \%$, and a requirement for peaks to be present in $>20 \%$ of the spectra. The peak intensities from the duplicate spectra from each patient were averaged and the resulting peak intensities of the 60 HCC patients and 84 nonHCC patients in the training set were compared by two-sample $t$-test and the area under the receiver operator characteristic (ROC) curve used to assess the discriminatory power of each peak.

\section{Sample classification}

Artificial neural networks (ANNs) were used to build committee models to classify serum samples into HCC and non-HCC groups using different numbers of significant peaks. The feed-forward neural networks consisted of three layers: an input layer, a hidden layer and an output layer. The number of input nodes was determined by the number of significant peaks from which the models were trained. The hidden layer connected the input and 
output layers, and the number of nodes in this layer controlled the complexity and performance of the neural networks. The output layer consisted of a single node whose output was used to classify sample status, representing HCC or non-HCC. The ANN had full connection from the input nodes to the hidden nodes and from the hidden nodes to the output node. All of the connection weights were randomly initialised in the range $(-1,+1)$. The ANNs were trained using the back propagation algorithm. In the procedure of training a committee model, a 10-fold cross-validation approach was used to reduce the risk of 'over fit' (Khan et al, 2001). The training data set was randomly partitioned into 10 subvalidation sets $(10 \%)$ and 10 subtraining sets (90\%). Each sample was contained only once in the subvalidation sets. Thus, 10 different ANNs were combined to create a committee model. A stepwise approach was used in which many committee models were built using various numbers of the most significant peaks. Significant peaks were identified by two-sample $t$-test if $P$ is less than 0.0123 (determined by a false discovery rate of 10\%) (Benjamini and Hochberg, 1995). The classification of the blind test set was made according to the majority decision of the six best committee models.

\section{Biomarker purification and identification}

Two pooled samples were prepared, one containing serum from five HCC patients with high SELDI intensity at $23 / 23.5 \mathrm{kDa}$ and one containing serum from five non-HCC patients with low SELDI intensity at $23 / 23.5 \mathrm{kDa}$. These two samples were diluted four-fold with $9 \mathrm{M}$ urea, $2 \%$ CHAPS, $50 \mathrm{~mm}$ Tris/ $\mathrm{HCl}$ ( $\mathrm{pH} 9.0)$ and applied to Q Ceramic HyperD F anion exchange resin. Proteins were eluted stepwise from the resin using buffers at $\mathrm{pH} 9,7,5,4,3$ and an organic wash. The proteins in these fractions were monitored by SELDI using $\mathrm{Cu}^{2+}$-loaded IMAC30 protein-chip arrays and the fractions containing the $23 / 23.5 \mathrm{kDa}$ biomarkers were applied to a monolithic C18 RP-HPLC column (BeckmanCoulter PF-2D system) and eluted with an acetonitrile gradient in $0.1 \%$ trifluoroacetic acid at a flow rate of $0.75 \mathrm{ml} \mathrm{min}^{-1}$. Fractions were collected $(0.6 \mathrm{~min})$ and analysed by SELDI on $\mathrm{Cu}^{2+}$-loaded IMAC30 protein-chip arrays. Fractions containing the 23/ $23.5 \mathrm{kDa}$ peak were concentrated and further purified by nonreducing $12 \%$ SDS - PAGE using MES running buffer (Invitrogen). The bands corresponding to the $23 / 23.5 \mathrm{kDa}$ biomarkers were excised and washed in $40 \mathrm{~mm}$ ammonium bicarbonate $/ 50 \%$ acetonitrile. The gel slices were then treated with $50 \mathrm{~mm}$ DTT in $40 \mathrm{~mm}$ ammonium bicarbonate $/ 10 \%$ acetonitrile $\left(1 \mathrm{~h}\right.$ at $\left.60^{\circ} \mathrm{C}\right)$ followed by $100 \mathrm{~mm}$ iodoacetamide $(30 \mathrm{~min}$ at room temperature in the dark). After several washes with $40 \mathrm{~mm}$ ammonium bicarbonate $/ 10 \%$ acetonitrile, $20 \mu \mathrm{l}$ of $12.5 \mu \mathrm{g} \mathrm{ml}^{-1}$ sequencing grade trypsin (Promega) was added to the dried gel bands and digestion allowed to proceed at $37^{\circ} \mathrm{C}$ overnight. Peptides were extracted with $100 \mu \mathrm{l}$ of $3 \%$ formic acid and analysed by LC-MS/MS using a ThermoFinnigan LCQ Deca XP Plus Ion-Trap linked directly to an LC Packings/Dionex Ultimate nanobore HPLC system. MS/MS data were searched against a database of nonredundant human protein sequences extracted from NCBI using SEQUEST. Data were filtered using Xcorr values of 1.5, 2 and 2.5 for singly, doubly and triply charged parent ions, respectively, and only first hits were considered.

\section{RESULTS}

\section{Surface-enhanced laser desorption/ionisation quality control}

Quality control samples were run in triplicate on all six bioprocessors. ANOVA analysis in Biomarker Wizard software provided no evidence of significant differences between the QC data from different bioprocessors. The coefficient of variation $(\mathrm{CV})$ of the 18 intensities measured for each proteomic feature was
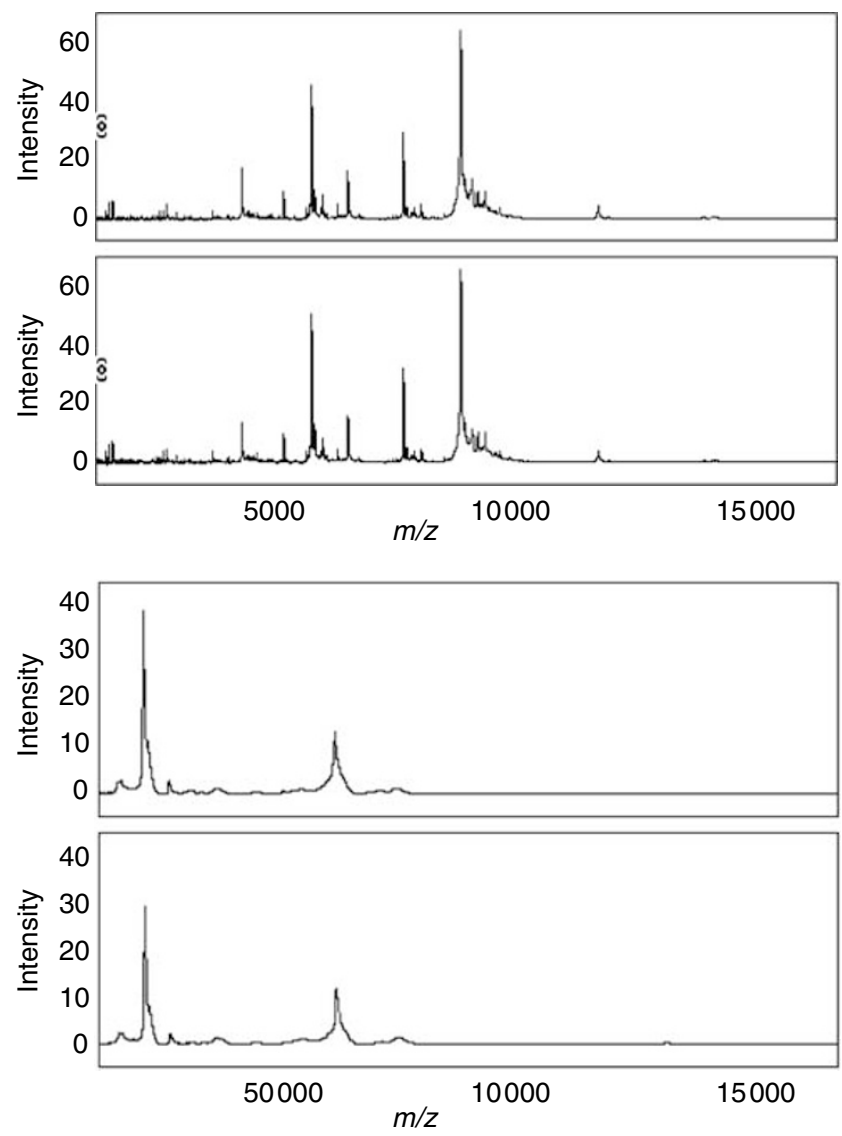

Figure I Duplicate low $(0-20000 \mathrm{~m} / \mathrm{z})$ and high $(20000-200000 \mathrm{~m} / \mathrm{z})$ spectra from a single HCC patient. The spectra were collected 13 days apart on different bioprocessors as described in the Materials and Methods section.

calculated and averaged across the 35 most intense peaks. This yielded an average CV of $20 \pm 8 \%$ (mean \pm s.d.) obtained during the course of the survey, consistent with the manufacturer's specification $(15-20 \%)$. Visual inspection of the data revealed no gross differences between duplicate spectra from each patient's serum samples and no data had to be discarded on this basis. An example of duplicate $0-20$ and $0-200 \mathrm{kDa}$ spectra for one HCC patient is shown in Figure 1.

A number of patient's samples were haemolysed giving rise to atypical spectra. These were characterised by high haemoglobin peaks (15.1 and $15.9 \mathrm{kDa})$ and/or a low albumin peak $(66.5 \mathrm{kDa})$. We discarded 34 samples from further data analysis on the basis of a SELDI intensity $>5$ at $15.9 \mathrm{kDa}$ or $<5$ at $66.5 \mathrm{kDa}$. The haemolysed samples were distributed evenly among the HCC and non-HCC patients.

\section{Significant differences between the serum of HCC and non- HCC patients}

Of the 138 peaks picked and clustered by the Biomarker Wizard software, 17 were significantly different at $P<0.0123$ (corresponding to a false discovery rate of $10 \%$ ) and these are shown in Table2. These peaks have areas under the ROC curve ranging from 0.58 to 0.71 indicating possible diagnostic utility, especially if several of these peaks could be used to build a classifier.

\section{Artificial neural networks}

A total of 17 ANN committee models were developed using up to 17 of the most significant peaks in the training set $(P<0.0123)$. The 
Table 2 Proteomic features most significantly different between HCC and non-HCC patients

\begin{tabular}{lccc}
\hline Peak (m/z) & $\boldsymbol{P}$ (t-test) & $\begin{array}{c}\text { Area under } \\
\text { ROC curve }\end{array}$ & $\begin{array}{c}\text { Ratio of mean } \\
\text { intensity of HCC } \\
\text { sera relative to } \\
\text { non-HCC sera }\end{array}$ \\
\hline 132200 & $1 \times 10^{-5}$ & 0.709 & 0.64 \\
23530 & $5 \times 10^{-5}$ & 0.708 & 1.50 \\
22960 & 0.0008 & 0.693 & 1.46 \\
53830 & 0.0024 & 0.619 & 1.43 \\
5254 & 0.0053 & 0.650 & 1.37 \\
33350 & 0.0055 & 0.634 & 0.90 \\
2362 & 0.0060 & 0.619 & 0.69 \\
3088 & 0.0064 & 0.614 & 0.59 \\
5811 & 0.0064 & 0.608 & 1.67 \\
10270 & 0.0072 & 0.584 & 0.72 \\
149410 & 0.0081 & 0.625 & 1.30 \\
66480 & 0.0084 & 0.630 & 0.90 \\
2273 & 0.0085 & 0.634 & 0.70 \\
94710 & 0.0088 & 0.641 & 0.85 \\
2792 & 0.0089 & 0.606 & 0.68 \\
4795 & 0.0119 & 0.608 & 1.26 \\
5826 & 0.0121 & 0.599 & 1.51 \\
\hline 14 & 0.512 & & \\
\hline
\end{tabular}

The table shows the 17 most significant proteomic features based on a two-sample test. For each feature, we show the mass/charge $(\mathrm{m} / \mathrm{z})$ ratio, $P$-value, area under the receiver operator characteristic (ROC) curve and the mean intensity in the HCC patients relative to the non-HCC patients.

best performing committee models were selected by their ability to correctly assign samples as HCC or non-HCC by 10 -fold crossvalidation of the training set. A total of 170 ANNs were trained (10 for each committee model). The committee models using the most significant $4,7,10,11,15$ and 17 features were selected with average misclassification rates of $10-15 \%$ (cross-validation). Rather than using individual committee models, the majority vote from these six committee models was used to classify the blind test set. It should be emphasised that the blind test set (the key to which was held exclusively by the Bondy group) was only unblinded when the classification model had been finalised, hence operator bias can be excluded from the success of the ANN classification.

The blind test set consisted of 17 HCC and 21 non-HCC patients. The majority vote of our ANN committee models correctly predicted 16 HCC patients and 18 non-HCC patients giving $94 \%$ sensitivity (95\% confidence interval $73-99 \%$, calculated according to Wilson, 1927) and $86 \%$ specificity (95\% confidence interval $65-$ 95\%). Interestingly, all $10 \mathrm{HCC}$ patients in the blind test set that had tumours less than $30 \mathrm{~mm}$ were correctly identified, indicating that SELDI can detect liver tumours at an early stage. One of the non-HCC patients diagnosed as having HCC by our analytical approach developed a $25 \mathrm{~mm}$ diameter tumour within 6 months of the sample being taken. The area under the ROC curve for the ANN prediction of the blind test set was 0.92 (Figure 2) comparing favourably with 0.73 for AFP (calculated across the whole study).

\section{Biomarker identification}

We selected a broad proteomic feature with peaks at $\mathrm{m} / \mathrm{z}$ ratios of 22960 and 23530 (hereafter referred to as the '23/23.5 kDa peak') that was significantly elevated in the serum of HCC patients compared to those with cirrhosis alone as a suitable candidate for purification and identification. Of the 138 SELDI peaks, these two were the best discriminators for HCC in this cohort of patients with the exception of a peak with an $\mathrm{m} / z$ ratio of 132200 . Although not formally identified, this peak copurifies with albumin and most likely represents a dimer of albumin. The $23 / 23.5 \mathrm{kDa}$ peak was purified in parallel from a pool of HCC sera with high intensity at

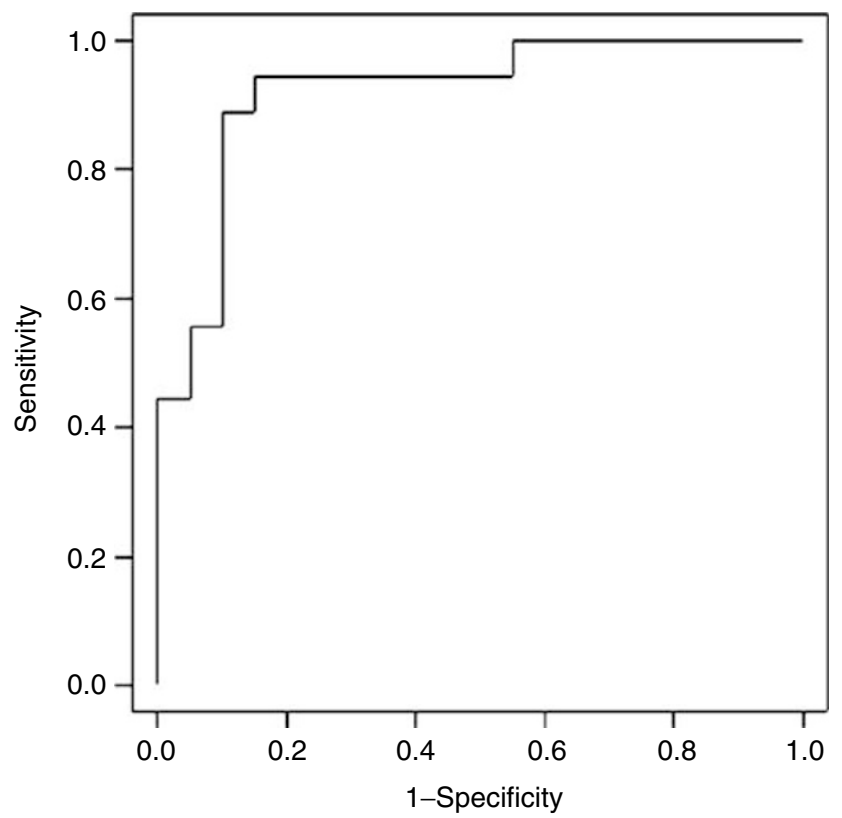

Figure 2 Receiver operator characteristic curve for the ANN classificication of samples in the blind test set.

$23 / 23.5 \mathrm{kDa}$ and a pool of non-HCC sera with low intensity at 23/ $23.5 \mathrm{kDa}$ (Figure 3). These sera were denatured with urea at $\mathrm{pH} 9$ and applied to anion exchange resin. A double peak at $23 / 23.5 \mathrm{kDa}$ was found in the resin flow-through (' $\mathrm{pH} 9$ fraction') from the 'high' sample but was less intense in the $\mathrm{pH} 9$ fraction from the 'low' sample indicating that our biomarker is a basic protein that does not bind to the resin under these conditions. The two $\mathrm{pH} 9$ fractions, enriched in the $23 / 23.5 \mathrm{kDa}$ peak, were applied to an RPHPLC column and proteins eluted with an acetonitrile gradient. The $23 / 23.5 \mathrm{kDa}$ peak was detected by SELDI in fractions corresponding to $60-70 \%$ acetonitrile and again was more intense in the 'high' sample (Figure 3). The fractions containing the 23/ $23.5 \mathrm{kDa}$ peak were concentrated by centrifugal evaporation and the proteins separated by SDS-PAGE (Figure 4). A band with $\sim 23 \mathrm{kDa}$ mobility that was more intensely stained in the 'high' sample (Figure 4) was excised and trypsinised.

LC-MS/MS identified 34 tryptic peptides of immunoglobulin $\kappa$ light chain and eight peptides from immunoglobulin $\lambda$ light chain. Several homologous peptides, each with a unique mass but reflecting the same part of the sequence of different $\kappa$ or $\lambda$ light chains were seen. For example, 12 homologous peptides were found corresponding to the N-terminal tryptic fragment of $\kappa$ light chain. The data are summarised in Table 3. For each of the seven sets of homologous peptides for the $\kappa$ light chain and five for the $\lambda$ light chain, we have provided the sequence with the highest Xcorr. The peptides listed in Table 3 cover 55 and $34 \%$ of the sequence of $\kappa$ and $\lambda$ light chains, respectively, assuming a polypeptide length of 215 amino acids. The multiplicity of peptides demonstrates that the $23 / 23.5 \mathrm{kDa}$ peak represents a diverse repertoire of immunoglobulin light chains, consistent with both the broad elution from the RP-HPLC column and the broad peak(s) in the SELDI spectra. The identity of the $23 / 23.5 \mathrm{kDa}$ biomarker was confirmed using an anti-human IgG polyclonal antibody to probe a blot of a SDS-PAGE gel of samples with high and low intensities of the biomarker (Figure 5). The anti-IgG antibody detects more immunoglobulin protein with an electrophoretic mobility of $20-30 \mathrm{kDa}$ (light chains) in the serum samples with greater SELDI intensity at $23 / 23.5 \mathrm{kDa}$ (Figure 4 ). The anti-IgG antibody also showed increased binding to proteins of 50 and $100-200 \mathrm{kDa}$ in 
HCC (high) sample
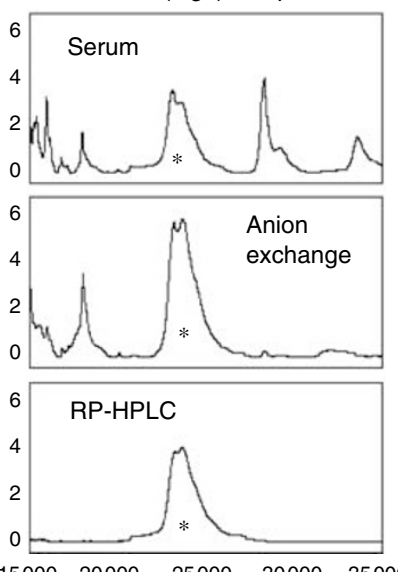

Non-HCC (low) sample
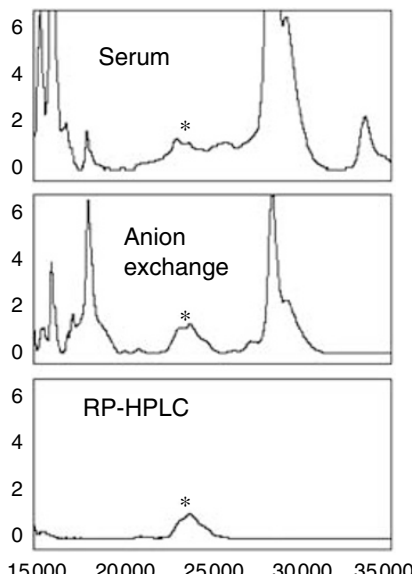

Figure 3 SELDI spectra following the 23/23.5 kDa biomarker purification. Pooled HCC and non-HCC sera, 'high' and 'low' in the biomarker, respectively, were purified in parallel. Spectra for the original pooled sera, the $\mathrm{pH} 9$ fraction from the anion exchange separation and the peak fraction from the RP-HPLC separation (fraction 25, $0.6 \mathrm{~min}$ ) are shown. *Indicates the $23 / 23.5 \mathrm{kDa}$ peak. All spectra were collected using $\mathrm{Cu}{ }^{2+}$-loaded IMAC chips.

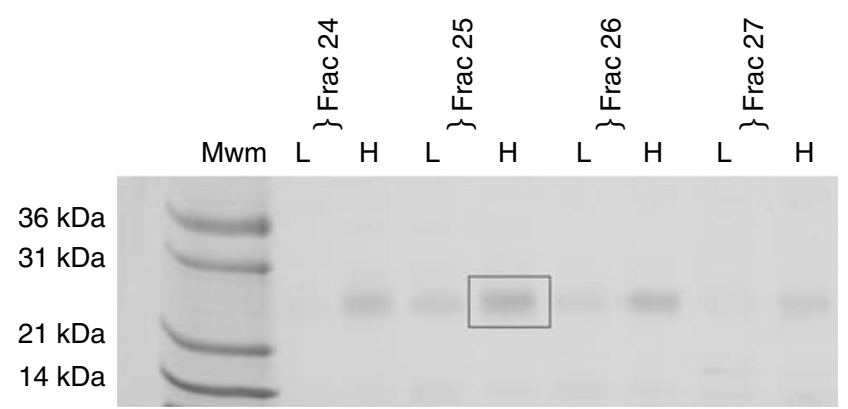

Figure 4 Coomassie stained I2\% SDS-PAGE of fractions 24-27 (each $0.6 \mathrm{~min}$ ) of the RP-HPLC of pooled HCC sera high $(\mathrm{H})$ and pooled non$\mathrm{HCC}$ sera low (L) in the 23/23.5 kDa biomarker. Mwm = molecular weight markers. The rectangle encloses the band that provided the tryptic peptides listed in Table 3.

the samples with greater SELDI intensity at $23 / 23.5 \mathrm{kDa}$ (data not shown). This suggests that there is an overall increase in the level of IgG in the serum of HCC patients. It is possible that the SELDI peaks at 54 and $149 \mathrm{kDa}$, upregulated in HCC patients (Table 2), represent immunoglobulin heavy chains and intact IgG, respectively.

\section{DISCUSSION}

In this study, we have shown that SELDI spectra are reproducible and capable of detecting differences in the serum proteome associated with HCC. Artificial neural networks developed using a training set of 84 non-HCC and 60 HCC patients were able to classify a blind test set of 21 non-HCC and 17 HCC patients with $94 \%$ sensitivity and $86 \%$ specificity. The accuracy of our ANNbased classification is far higher than the currently accepted biomarker, AFP (Gupta et al, 2003) (on the patient cohort used in this study, AFP $>20 \mathrm{ng} \mathrm{ml}^{-1}$ gave $46 \%$ sensitivity and $89 \%$ specificity). However, this is only a phase 1 biomarker discovery study (Pepe et al, 2001). Validation requires analysis of larger cohorts of patients collected at multiple sites. Some early SELDI-
Table 3 Peptides identified by LC-MS/MS from the tryptic digest of the 23/23.5 kDa biomarker

\begin{tabular}{|c|c|c|c|}
\hline Peptide sequence & Xcorr & $\begin{array}{c}\text { Number of } \\
\text { homologous } \\
\text { peptides }\end{array}$ & $\begin{array}{l}\text { Protein } \\
\text { identification }\end{array}$ \\
\hline EIVLTQSPATSLSPGER & 4.37 & 12 & Ig $\kappa$ variable region \\
\hline LLIYGASNLQTGVPSR & 2.95 & 9 & Ig $\kappa$ variable region \\
\hline FSGSNSGNTATLTISR & 3.94 & 5 & Ig $\kappa$ variable region \\
\hline TVAAPSVFIFPPSDEQLK & 4.01 & 3 & Ig $\kappa$ constant region \\
\hline SGTASWVLLNNFYPR & 4.79 & I & Ig $\kappa$ constant region \\
\hline DSTYSLSSTLTLSK & 3.67 & I & Ig $\kappa$ constant region \\
\hline VYACEVTHQGLSSPVTKSFNRG & 4.65 & 2 & Ig $\kappa$ constant region \\
\hline YVLTQPPSVSVAPGQTAR & 2.62 & 2 & $\lg \lambda$ variable region \\
\hline SGTSASLAISGLR & 3.91 & 1 & $\lg \lambda$ variable region \\
\hline LTVLSQPK & 2.52 & 3 & $\lg \lambda$ variable region \\
\hline YAASSYLSLTPEQWK & 2.95 & । & $\lg \lambda$ constant region \\
\hline SYSCQVTHEGSTVEK & 3.31 & I & $\lg \lambda$ constant region \\
\hline
\end{tabular}
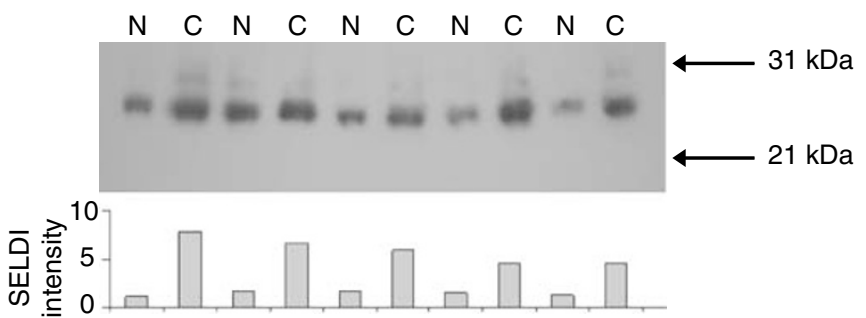

Figure 5 Anti-human IgG blot. Proteins were separated by SDS-PAGE on a $4-12 \%$ slab gel and blotted on to PVDF membrane. The blot was probed using horseradish peroxidase-conjugated rabbit polyclonal antihuman $\lg G$ antibody (Abcam). The blot shows alternating lanes of nonHCC (N) and HCC (C) sera. The histogram shows the SELDI intensity of each sample at $\mathrm{m} / \mathrm{z} 22960$.

based biomarker studies have been heavily criticised for potential bias in their design. This may arise from collection of the cancer and noncancer sera in a different manner, differing storage conditions or poor experimental design, for example, temporal separation between the analysis of the cancer and noncancer samples. In addition, the small sample numbers and highdimensionality of the data require that care is taken not to overfit data. We have addressed these issues in our experimental design: QC samples were run on all bioprocessors to exclude trends in the protein-chip array reader's performance, block randomisation ensured that any variations in chip quality did not bias the study between HCC and non-HCC or between training and validation sets and the proteomic team in the UK that analysed the samples were unaware of the identities of the blind test set: the 'key' was held in France until the patient classification had been completed. In addition, the patient groups were well matched with regard to age (Table 1) and the male/female ratio was well balanced in the test set. Although the male/female ratio was not as well balanced in the training set, only two of the 17 peaks used to build ANNs were significantly different between male and female patients $(\mathrm{m} / \mathrm{z} 4795$ and $66480, P<0.05)$. Therefore, we can be confident that the proteomic changes we have identified are related to HCC. They could be either proteins secreted by the tumour or arising from secretion from the tumors, induced by inflammatory or immunological response to the tumour or the hallmark of predisposing factors to HCC occurrence. The $23 / 23.5 \mathrm{kDa}$ peak that we have identified as immunoglobulin light chains may fall into the latter category: IgG levels are higher in patients with more advanced chronic liver disease, itself a predictive factor for HCC. 
Previous reports have provided evidence that HCC produces changes in the serum proteome of chronic liver disease patients that can be detected by SELDI (Poon et al, 2003; Paradis et al, 2005; Schwegler et al, 2005). Schwegler et al (2005) used SELDI to analyse the serum of 50 hepatitis $\mathrm{C}$ patients (28 with HCC). They achieved $61 \%$ sensitivity and $76 \%$ specificity (using decision trees) and found peaks at 5.8 and $11.7 \mathrm{kDa}$ elevated in HCC. We also see a peak at $5.8 \mathrm{kDa}$ that is upregulated in HCC. Paradis et al (2005) were able to classify a set of 82 cirrhotic patients (38 with HCC) with $90 \%$ accuracy using logistic regression of data from $\mathrm{Zn}^{2+}$ loaded IMAC protein-chip arrays and published a list of 30 proteomic features significantly different between HCC and non-HCC patients. Paradis et al did not observe our $23 / 23.5 \mathrm{kDa}$ biomarker, but some discriminatory features are common to both studies: the intensity of peaks at 33.2 and 66.4 and $102 \mathrm{kDa}$ are decreased (66.4 and $33.2 \mathrm{kDa}$ presumably representing singly and doubly charged ions of albumin). The use of $\mathrm{Zn}^{2+}$ rather than $\mathrm{Cu}^{2+}$ as the chromatographic ligand and differences in sample preparation and Protein-chip reader settings may account for some differences between the studies in addition to different underlying causes of chronic liver disease and stage of HCC progression. The discriminatory peaks in this study also differ from our earlier work in which serum samples were fractionated prior to SELDI (Poon et al, 2003) and again this may be due to the greater mean age and earlier stage of HCC progression in the patients used in our current work. Additionally, the fractionation may have unmasked better discriminators than those observed with whole serum.

It is possible that the improved accuracy of immunoassays over SELDI 'quantitation' could improve the performance of biomarkers originally identified by SELDI. The use of multiple carefully chosen markers should enhance both the sensitivity and specificity of HCC screening. Interestingly, unpublished pilot SELDI studies in our laboratory using $\mathrm{Cu}^{2+}$-loaded IMAC30 protein-chip arrays also indicate a $23 / 23.5 \mathrm{kDa}$ peak that is elevated by $\sim 20 \%$ in the serum of colorectal cancer patients (with respect to healthy controls), but not in the serum of oral, breast or prostate cancer patients.

\section{ACKNOWLEDGEMENTS}

This work was funded by in part by Cancer Research UK and the Tony Turnbull Memorial Fund.

\section{REFERENCES}

Adam B-L, Qu YQ, Davis JW, Ward MD, Clements MA, Cazares LH, Semmes OJ, Schellhammer PF, Yasui Y, Feng Z, Wright Jr GL (2002) Serum protein fingerprinting coupled with a pattern-matching algorithm distinguishes prostate cancer from benign prostate hyperplasia and healthy men. Cancer Res 62: 3609-3614

Baggerly KA, Morris JS, Coombes KR (2004) Reproducibility of SELDI-TOF protein patterns in serum: comparing data sets from different experiments. Bioinformatics 20: 777-785

Beaugrand M, N'kontchou G, Seror O, Ganne N, Trinchet J-C (2005) Local/ regional and systemic treatments of hepatocellular carcinoma. Semin Liver Dis 205: 201-211

Benjamini Y, Hochberg Y (1995) Controlling the false discovery rate: a practical and powerful approach to multiple testing. J Roy Statist Soc Ser $B$ (Methodol) 57: $289-300$

Bruix J, Sherman M, Llovet JM, Beaugrand M, Lencioni R, Burroughs AK, Christensen E, Pagliaro L, Colombo M, Rodes J (2001) EASL panel of experts on HCC. Clinical management of hepatocellular carcinoma. Conclusions of the Barcelona-2000 EASL conference. J Hepatol 35: $421-430$

Chang MH, Chen CJ, Lai MS, Hsu HM, Wu TC, Kong MS, Liang DC, Shau WY, Chen CS (1997) Universal hepatitis B vaccination in Taiwan and the incidence of hepatocellular carcinoma in children. Taiwan childhood hepatoma study group. N Engl J Med 336: 1855-1859

Chen Y, Zheng S, Yu J-K, Hu X (2004) Artificial neural networks analysis of surface-enhanced laser desorbtion/ionisation mass spectra of serum protein pattern distinguishes colorectal cancer from healthy population. Clin Cancer Res 10: 8380 - 8385

Diamandis EP (2004) Mass spectrometry as a diagnostic and a cancer biomarker discovery tool. Mol Cell Proteom 3: 367-378

Di Bisceglie AM, Order SE, Klein JL, Waggoner JG, Sjogren MH, Kuo G, Houghton M, Choo Q-L, Hoofnagle JH (1991) The role of chronic viral hepatitis in hepatocellular carcinoma in the United States. Am J Gastroenterol 86: $335-338$

El-Sarag HB, Hashem B (2004) Hepatocellular carcinoma: an epidemiologic view. J Clin Gastroenterol 35: S72-S78

Gupta S, Bent S, Kohlwes J (2003) Test characteristic of alpha-fetoprotein for detecting hepatocellular carcinoma in patients with heaptitis C. A systematic review and critical analysis. Ann Internal Med 139: 46- 50

Johnson PJ (2001) The role of serum alpha-fetoprotein estimation in the diagnosis and management of hepatocellular carcinoma. In: Clinics in Liver Disease, Di Bisceglie A (ed) Vol. 5, pp 145-159. Philadelphia: WB Saunders
Khan J, Wei WS, Ringner M, Saal LH, Ladanyi M, Westerman F, Berthold F, Schwab M, Antonescu C, Peterson C, Meltzer PS (2001) Classification and diagnostic prediction of cancers using gene expression profiling and artificial neural networks. Nat Med 7: 673-679

Li J, Rosenzweig JM, Wang YY, Chan DW (2002) Proteomics and bioinformatics approaches for identification of serum biomarkers to detect breast cancer. Clin Chem 48: 1296-1304

Mazzaferro V, Regalia E, Doci R, Andreola S, Pulvirenti A (1996) Liver transplantation for treatment of small hepatocellular carcinomas in patients with cirrhosis. N Engl J Med 334: 693-699

Paradis V, Degos F, Dargere D, Pham N, Belghiti J, Degott C, Janeau J-L, Bezeaud A, Delforge D, Cubizolles M, Laurendeau I, Bedossa P (2005) Identification of a new marker of hepatocellular carcinoma by serum protein profiling of patients with chronic liver disease. Hepatology 41: 40-47

Parkin DM, Bray F, Ferlay J, Pisani P (2001) Estimating the world cancer burden: Globocan 2000. Int J Cancer 94: 153-156

Pepe MS, Etzioni R, Feng Z, Potter JD, Thompson ML, Thornquist M, Winget M, Yasui Y (2001) Phases of biomarker development for early detection of cancer. J Natl Cancer Instit 93: 1054-1061

Poon TCW, Yip T-T, Chan ATC, Yip C, Yip V, Mok TSK, Lee CCY, Leung TWT, Ho SKW, Johnson PJ (2003) Comprehensive proteomic profiling identifies serum proteomic signatures for detection of hepatocellular carcinoma and its subtypes. Clin Chem 49: $752-760$

Ransohoff DF (2004) Rules of evidence for cancer molecular-marker discovery and validation. Nat Rev: Cancer 4: 309-313

Schwegler EE, Cazares L, Steel LF, Adam B-L, Johnson DA, Semmes OJ, Block TM, Marrero JA, Drake RR (2005) SELDI-TOF MS profiling of serum for detection of the progression of chronic hepatitis $\mathrm{C}$ to hepatocellular carcinoma. Hepatology 41: 634-642

Sherman M (2005) Screening for hepatocellular carcinoma. Best Practice Res Clin Gastroenterol 19: $101-118$

Trevisani F, De NS, Rappaccini G, Farinati F, Benvegnu L, Zoli M, Grazi GL, Del PP, Di N, Bernardi M (2002) Semiannual and annual surveillance of cirrhotic patients for hepatocellular carcinoma: effects on cancer stage and patient survival (Italian experience). Am J Gastroenterol 97: 734-744

Wilson EB (1927) Probable inference, the law of succession, and statistical inference. J Am Statist Assoc 22: 209-212

Zhang Z, Bast Jr B, Yu Y, Li J, Sokoll LJ, Rai AJ, Rosenzweig JM, Cameron B, Wang YY, Meng X-Y, Berchuk A, Haaftenday CV, Hacker NF, Bruijn HWA, Zee AGVD, Jacobs IJ, Fung ET, Chan DW (2004) Three biomarkers identified from serum proteomic analysis for the detection of early stage ovarian cancer. Cancer Res 64: 5882-5890 